OPEN ACCESS

Edited by:

Antonio Pisani,

University of Rome Tor Vergata, Italy

Reviewed by:

Julia Muellner,

Bern University Hospital, Switzerland

Christian Schlenstedt,

University of Kiel, Germany

*Correspondence:

Christian Geroin

christian.geroin@univr.it

Michele Tinazzi

michele.tinazzi@univr.it

${ }^{\dagger}$ These authors have contributed equally to this work

Specialty section

This article was submitted to Movement Disorders,

a section of the journal

Frontiers in Neurology

Received: 22 November 2019 Accepted: 09 March 2020

Published: 31 March 2020

Citation:

Geroin C, Artusi CA, Gandolfi M,

Zanolin E, Ceravolo R, Capecci $M$ Andrenelli E, Ceravolo MG, Bonanni L,

Onofrj M, Telese R, Bellavita G,

Catalan M, Manganotti $P$,

Mazzucchi S, Giannoni S, Vacca L,

Stocchi F, Casali M,

Falup-Pecurariu C, Zibetti M,

Fasano A, Lopiano $L$ and Tinazzi $M$

(2020) Does the Degree of Trunk Bending Predict Patient Disability, Motor Impairment, Falls, and Back

Pain in Parkinson's Disease?

Front. Neurol. 11:207.

doi: 10.3389/fneur.2020.00207

\section{Does the Degree of Trunk Bending Predict Patient Disability, Motor Impairment, Falls, and Back Pain in Parkinson's Disease?}

Christian Geroin ${ }^{1 *}$, Carlo Alberto Artusi ${ }^{2 \dagger}$, Marialuisa Gandolfi ${ }^{3,4}$, Elisabetta Zanolin ${ }^{5}$, Roberto Ceravolo ${ }^{6}$, Marianna Capecci ${ }^{7}$, Elisa Andrenelli ${ }^{7}$, Maria Gabriella Ceravolo ${ }^{7}$, Laura Bonanni ${ }^{8}$, Marco Onofrj ${ }^{8}$, Roberta Telese ${ }^{8}$, Giulia Bellavita ${ }^{9}$, Mauro Catalan $^{9}$, Paolo Manganotti ${ }^{9}$, Sonia Mazzucchi ${ }^{6}$, Sara Giannoni ${ }^{6}$, Laura Vacca ${ }^{10}$, Fabrizio Stocchi ${ }^{10}$, Miriam Casali ${ }^{10}$, Cristian Falup-Pecurariu ${ }^{11}$, Maurizio Zibetti ${ }^{2}$, Alfonso Fasano ${ }^{12,13}$, Leonardo Lopiano $^{2}$ and Michele Tinazzi ${ }^{1 *}$

${ }^{1}$ Neurology Unit, Movement Disorders Division, Department of Neurosciences, Biomedicine and Movement Sciences, University of Verona, Verona, Italy, ${ }^{2}$ Department of Neuroscience "Rita Levi Montalcini", University of Torino, Torino, Italy, ${ }^{3}$ Neuromotor and Cognitive Rehabilitation Research Center, Department of Neurosciences, Biomedicine and Movement Sciences, University of Verona, Verona, Italy, ${ }^{4}$ Neurorehabilitation Unit, Azienda Ospedaliera Universitaria Integrata, Verona, Italy, ${ }^{5}$ Department of Public Health and Community Medicine, University and Hospital Trust of Verona, Verona, Italy, ${ }^{6}$ Department of Clinical and Experimental Medicine, University of Pisa, Pisa, Italy, ${ }^{7}$ Department of Experimental and Clinical Medicine, Neurorehabilitation Clinic, "Politecnica delle Marche" University, Ancona, Italy, ${ }^{8}$ Department of Neuroscience, Imaging and Clinical Sciences, University G.d'Annunzio of Chieti-Pescara, Chieti, Italy, ${ }^{9}$ Clinical Neurology Unit, Department of Medical, Surgical and Health Services, University of Trieste, Trieste, Italy, ${ }^{10}$ University and Institute for Research and Medical Care IRCCS San Raffaele, Rome, Italy, " ${ }^{11}$ Department of Neurology, Faculty of Medicine, Transilvania University, Brasov, Romania, ${ }^{12}$ Edmond J. Safra Program in Parkinson's Disease and the Morton and Gloria Shulman Movement Disorders Clinic, Division of Neurology, Toronto Western Hospital, UHN, University of Toronto, Toronto, ON, Canada,

${ }^{13}$ Krembil Brain Institute, Toronto, ON, Canada

Background: Postural abnormalities in Parkinson's disease (PD) form a spectrum of functional trunk misalignment, ranging from a "typical" parkinsonian stooped posture to progressively greater degrees of spine deviation.

Objective: To analyze the association between degree of postural abnormalities and disability and to determine cut-off values of trunk bending associated with limitations in activities of daily living (ADLs), motor impairment, falls, and back pain.

Methods: The study population was $283 \mathrm{PD}$ patients with $\geq 5^{\circ}$ of forward trunk bending (FTB), lateral trunk bending (LTB) or forward neck bending (FNB). The degrees were calculated using a wall goniometer (WG) and software-based measurements (SBM). Logistic regression models were used to identify the degree of bending associated with moderate/severe limitation in ADLs (Movement Disorders Society Unified PD Rating Scale [MDS-UPDRS] part $\| \geq 17$ ), moderate/severe motor impairment (MDS-UPDRS part III $\geq 33$ ), history of falls $(\geq 1)$, and moderate/severe back pain intensity (numeric rating scale $\geq 4$ ). The optimal cut-off was identified using receiver operating characteristic (ROC) curves.

Results: We found significant associations between modified Hoehn \& Yahr stage, disease duration, sex, and limitation in ADLs, motor impairment, back pain intensity, and history of falls. Degree of trunk bending was associated only with motor impairment 
in LTB (odds ratio [OR] 1.12; 95\% confidence interval [Cl], 1.03-1.22). ROC curves showed that patients with LTB of $10.5^{\circ}$ (SBM, AUC 0.626) may have moderate/severe motor impairment.

Conclusions: The severity of trunk misalignment does not fully explain limitation in ADLs, motor impairment, falls, and back pain. Multiple factors possibly related to an aggressive PD phenotype may account for disability in PD patients with FTB, LTB, and FNB.

Keywords: Parkinson's disease, camptocormia, Pisa syndrome, anterocollis, postural abnormalities

\section{INTRODUCTION}

Postural abnormalities are common features of Parkinson's disease (PD) and manifest in over $20 \%$ of patients during the course of disease (1). Three main types of PD-associated postural abnormalities are distinguished: camptocormia (CC), defined as an involuntary forward trunk bending (FTB) appearing during standing or walking and resolving in a supine position of at least $30^{\circ}$ at the lumbar fulcrum and/or at least $45^{\circ}$ at the thoracic fulcrum (2); Pisa syndrome (PS), defined as a sustained lateral trunk bending (LTB) of at least $10^{\circ}$ worsened by prolonged sitting or walking (3); and anterocollis (AC), defined as an involuntary forward neck bending (FNB) of at least $45^{\circ}$ (3). PD studies investigating CC, PS, and AC report greater disability in patients with postural abnormalities and a higher risk of falls, back pain, and worse quality of life (1, 3-6).

However, PD-associated postural abnormalities form a spectrum of functional trunk misalignment, ranging from a "typical" parkinsonian stooped posture, with rounding of the shoulders and flexion of the hips and knees, to progressively greater degrees of trunk bending. The current criteria for the definition of CC, PS, and AC are mainly based on expert opinion (3) or expert consensus (2), leaving issues open on the classification of postural abnormalities not fulfilling the criteria for CC, PS, or AC. Given the lack of a common and comprehensive classification for postural abnormalities and the still unexplored impact of "milder" trunk flexion on disability, there is a rationale for revision of the classification of $\mathrm{PD}$-associated postural abnormalities: (1) criteria for CC, PS, and AC have been decided a priori based on observational judgment of movement disorder experts and are not anchored to any formal measure of activities of daily living (ADLs), motor impairment, risk of falls or pain; and (2) the current cut-offs refer to very severe conditions, leaving many PD patients with potentially disabling postural abnormalities without an effective diagnosis of postural abnormalities.

In this study, our primary aim was to analyze the association between the degree of postural abnormalities and disability, expressed as limitation in ADLs, motor impairment, falls, and back pain. The secondary aim was to determine the cut-off values of trunk/neck bending that best predict the disability. We hypothesized that significant disability in patients with postural abnormalities may not be entirely related to the degrees of trunk bending and that disability may occur at lower degrees of trunk bending than the standard criteria for defining CC, PS, and AC, which identify a final stage of a complex process of trunk control impairment.

\section{METHODS}

For this study we retrieved data from a database created for a multicenter epidemiological study on postural abnormalities in $\mathrm{PD}$, encompassing comprehensive information on 811 consecutive outpatients with $\mathrm{PD}$ attending seven tertiary movement disorder centers in Italy (1).

\section{Participants}

The study population was patients with a diagnosis of idiopathic PD (7) presenting $\geq 5^{\circ}$ of FTB, LTB or FNB as measured with a wall goniometer. Exclusion criteria were: concomitant neurological diseases known to negatively affect posture, previous major spinal surgery, skeletal, or muscle disease (i.e., vertebral fractures, spondylodiscitis, and inflammatory myopathy), and treatment with medications possibly causing posture alterations (i.e., neuroleptics and antiemetics, except for clozapine, quetiapine, and domperidone) in the 6 months prior to enrollment.

The institutional review boards of the participating centers reviewed and approved the study protocol. All patients were informed about the nature of the study and gave their written consent to participate. Authorization was obtained for disclosure (consent-to-disclose) of any recognizable persons in photographs. The study was registered at https://clinicaltrials.gov (NCT03573232).

\section{Procedures}

At each center, patients were evaluated while on their usual drug treatment during the ON phase. Postural evaluation was performed with the use of a wall goniometer (WG) and analysis of patients' photographs during a single session by the same rater assigned before the start of the study. The following clinical and demographical features were recorded: age, gender, age at PD onset, Movement Disorders Society Unified Parkinson's Disease Rating Scale (MDS-UPDRS) part I-IV to assess PD severity (8), modified Hoehn \& Yahr (H\&Y) scale to assess PD stage, disease duration (in years since diagnosis), and PD phenotype (rigid-akinetic, tremor-dominant, or mixed type) (9). 


\section{Outcome Measures}

\section{Evaluation of Postural Abnormalities}

Two instruments were used to measure FTB, LTB, and FNB. The degree of trunk bending was calculated using the WG $(2,10,11)$ and software-based measurements (SBM) with the freeware program Kinovea ${ }^{\circledR}(10,12)$. Patients with FTB were subdivided into a lower and an upper FTB angle group according to the fulcrum level (2). For the WG measures, a rater trained at each center rated the angles independently. For SBM, an experienced rater (C.G.) was trained in the use of the freeware program Kinovea ${ }^{\circledR}$ and performed all measurements on the patients' photographs. Interobserver reliability between the two raters in the use of WG and softwarebased measurement to measure FTB, LTB, and FNB is good to excellent (10).

The WG was used to visually estimate the degree of bending in patients while standing or sitting $(10,11)$. The zero of WG was positioned at the level of the fulcrum. A line perpendicular to the ground and an imaginary line drawn from the fulcrum of bending through the $\mathrm{C} 7$ spinal process (or the tragus of the ear for the FNB) formed the outer angle. The minimal detectable change for the $W G$ recorded in degrees was $5^{\circ}$.

All patients underwent evaluation also with SBM. The lower FTB angle was calculated on the sagittal view photographs using the software-based malleolus method. A line was drawn from the L5 end of the L5/C7 line to the lateral malleolus of the foot, the external angle between these lines was measured (Figure 1A) $(10,13)$. The upper FTB angle was calculated on the sagittal view photographs as the outer angle between the two lines fulcrumL5 and fulcrum-C7, with the fulcrum defined as the most distant point perpendicular to the L5/C7 line (Figure 1B) $(10,12)$. The LTB angle was calculated on the planar view photographs as the angle between (a) the vertical axis and (b) a line connecting the fulcrum of the bent spine with the $\mathrm{C} 7$ spinous process (Figure 1C) (14). The FNB was calculated on the sagittal view photographs as the angle between (a) a line connecting the C7 spinous process and the tragus of the ear, and (b) the line perpendicular to the ground (Figure 1D) (15).

\section{Evaluation of Limitation in ADLs, Motor Impairment, Falls, and Pain}

Limitation in ADLs was evaluated according to the MDS-UPDRS part II score (8) and motor impairment by the MDS-UPDRS part III score (8). According to the literature, an MDS-UPDRS part II score $\geq 17$ identifies moderate/severe limitation in $\operatorname{ADLs}(16,17)$ and an MDS-UPDRS part III score $\geq 33$ moderate/severe motor

TABLE 1 | Clinical Features of PD Patients with one or more postural abnormality of forward trunk bending, lateral trunk bending, and forward neck bending.

\begin{tabular}{|c|c|c|c|}
\hline \multirow[t]{2}{*}{ Variables } & \multicolumn{3}{|c|}{ Patients with one or more PA } \\
\hline & FTB & LTB & FNB \\
\hline No. of patients & 215 & 88 & 61 \\
\hline Age, mean (SD), y & $73.50(8.1)$ & $73.23(7)$ & $70.97(7.8)$ \\
\hline \multicolumn{4}{|l|}{ Gender, No. (\%), } \\
\hline Male & $134(62.3)$ & $51(58)$ & $43(70.5)$ \\
\hline Female & $81(37.7)$ & $37(42)$ & $18(29.5)$ \\
\hline Age at PD onset, mean (SD), y & $65.05(9.8)$ & $62.69(10.5)$ & $61.3(10.3)$ \\
\hline $\begin{array}{l}\text { UPDRS Total score on state, } \\
\text { mean (SD) }\end{array}$ & $56.9(24.6)$ & $58.02(27.7)$ & $62.8(29.1)$ \\
\hline I & $7.7(6.4)$ & $7.33(6.5)$ & $8.8(6.7)$ \\
\hline$\|$ & $15.3(7.9)$ & $17.7(8.9)$ & $15.6(9.3)$ \\
\hline III & $31.8(13.4)$ & $30.3(14)$ & $36(15.7)$ \\
\hline IV & $2(2.93)$ & $2.7(3.7)$ & $2.1(2.8)$ \\
\hline $\begin{array}{l}\text { Modified H\&Y stage, mean } \\
\text { (SD) }\end{array}$ & $2.62(0.8)$ & $3.09(0.9)$ & $2.5(0.9)$ \\
\hline Disease Duration, mean (SD), y & $8.02(5.9)$ & $10.22(7.1)$ & $9.5(7.6)$ \\
\hline \multicolumn{4}{|l|}{ Dominant Phenotype, $n$ (\%) } \\
\hline Bradykinetic/Rigid type & $106(49.3)$ & $47(53.4)$ & $26(42.6)$ \\
\hline Tremor type & $33(15.4)$ & $20(22.7)$ & $18(29.5)$ \\
\hline Mixed Type & 76 (35.3) & $21(23.9)$ & $17(27.9)$ \\
\hline $\begin{array}{l}\text { L-dopa equivalent daily dose, } \\
\text { mg, mean } \\
\text { (SD) }\end{array}$ & 632.27 (349.54) & 666.43 (339.76) & $581.13(330.23)$ \\
\hline
\end{tabular}

SD, standard deviation; PD, Parkinson's Disease; PA, Postural Abnormalities including forward trunk bending (FTB), lateral trunk bending (LTB), and forward neck bending (FNB); y, years; H\&Y, Hoehn and Yahr; UPDRS, Unified Parkinson's Disease Rating Scale.
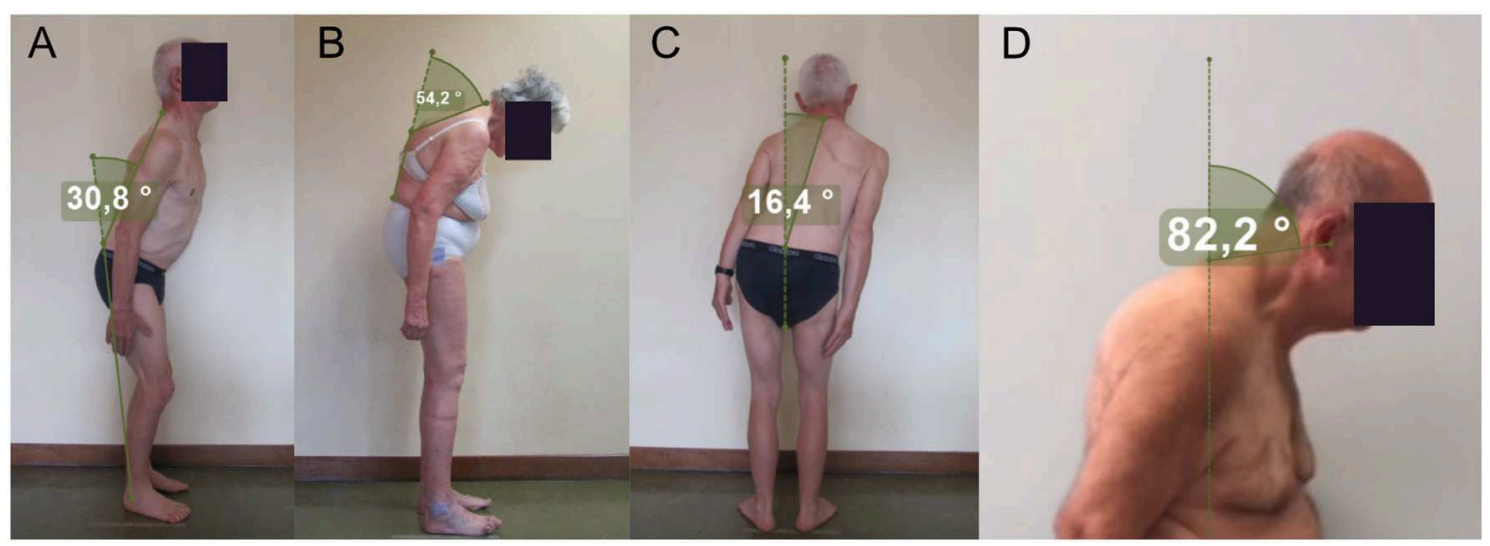

FIGURE 1 | (A) The malleolus method; (B) the upper method; (C,D) the perpendicular method. 
impairment (18). Patients were categorized as "fallers" if they reported having sustained at least one fall in the previous month (19). A numeric rating scale (NRS) graded from 0 (no pain at all) to 10 (excruciating pain) was used to rate back pain intensity (20). A score $\geq 4$ identified moderate/severe pain because it can interfere with the quality of life $(21,22)$.

\section{Statistical Analysis}

Descriptive statistics included frequency tables and calculation of means and standard deviation (SD) for each group of patients (FTB, LTB, and FNB). In patients with combined upper and lower FTB, we used the upper fulcrum degrees as upper FTB and the lower fulcrum degrees as lower FTB.

Logistic regression models were used to estimate unadjusted and adjusted odds ratios (95\% confidence interval [CI]) of MDS-UPDRS parts II, III, history of falls, NRS (dependent variables) in relation to the degree of trunk bending (independent variable); adjustment was performed taking into account the following sociodemographic and clinical features as covariates: gender, modified H\&Y stage, and disease duration. Variables having clinical relevance (1) or $p<0.05$ at univariate analysis were entered in the multivariate analysis as covariates.
When an association between the dependent variable and the degree of bending was found, sensitivity and specificity were calculated. Receiver operating characteristic (ROC) curves were constructed and the Youden index (the highest sum of values of sensitivity and specificity minus one) was calculated to obtain the optimal cut-off values of the degree of bending to identify patients with impairment. Areas under the curve (AUC) of the ROC curves were calculated to provide a measure of the overall discriminative ability of the prediction rule. Typically, a test with an AUC $>0.9$ has high accuracy, while an AUC $0.7-0.9$ indicates moderate accuracy, $0.5-0.7$ low accuracy, and 0.5 is compared to toss-up (chance result) (23). All tests were two-tailed and considered a $p$-value $<0.05$ as statistically significant. Data were analyzed using the Statistical Package for the Social Sciences (SPSS 22 for Mac, IBM-SPSS, Armonk, NY, USA).

\section{RESULTS}

\section{Clinical Features of Patients}

A total of 283 PD patients were enrolled according to the inclusion and exclusion criteria. A total of 215 patients presented FTB (175 upper type, 27 lower type, and 13 both types), 88

TABLE 2 | Clinical and demographic variables associated with upper FTB, as measured with a wall goniometer.

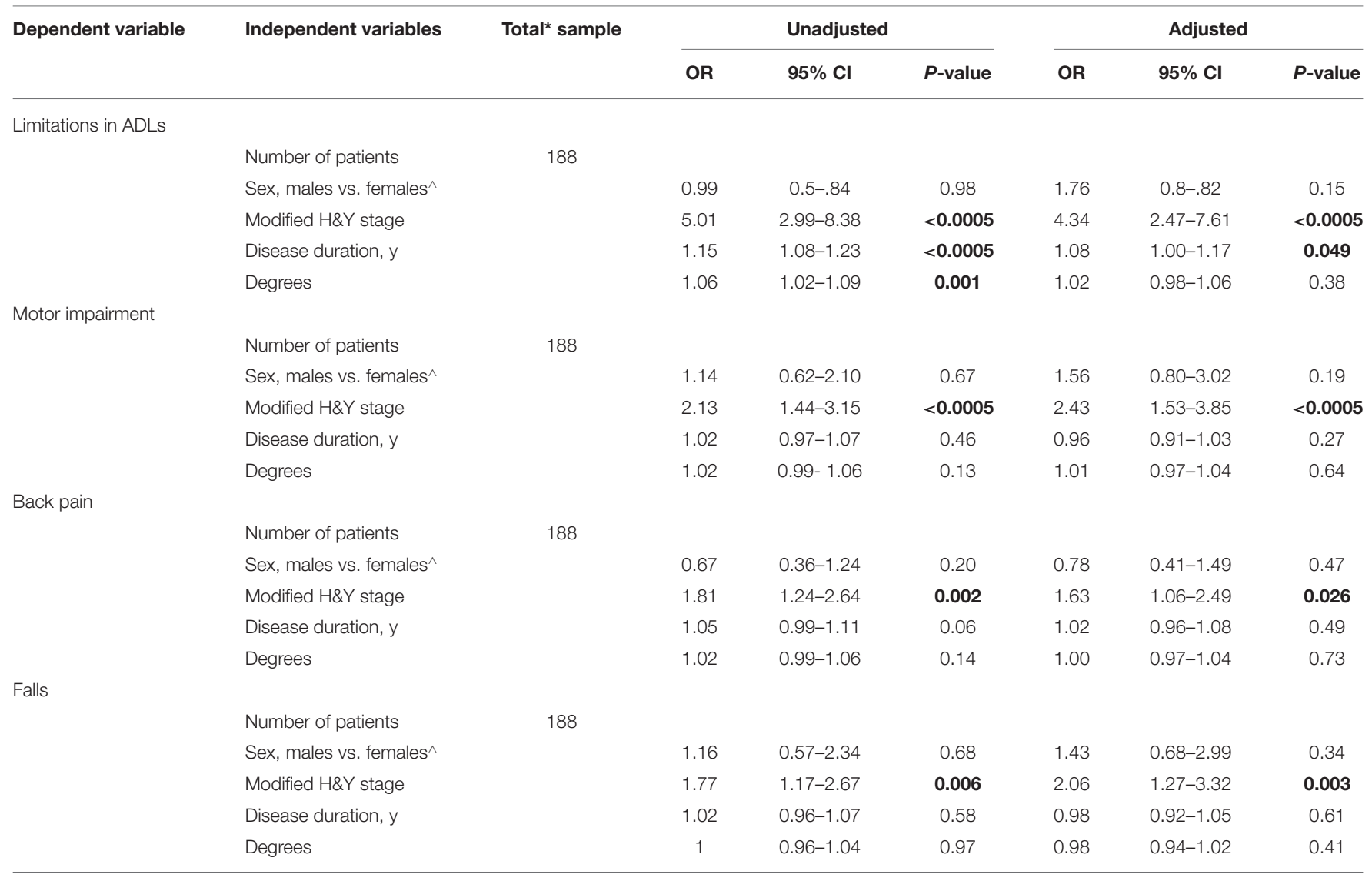

Cl, confidence interval; H\&Y, Hoehn and Yahr; OR, Odds Ratio; PAD, Parkinson disease; $\wedge$, denotes the reference category; FTB, forward trunk bending; "The total sample included the isolated upper FTB $(n=175)$ and combined forms (upper + lower FTB, $n=13)$; significant associations in bold at $p<0.05$. 
TABLE 3 | Clinical and demographic variables associated with lower FTB, as measured with a wall goniometer.

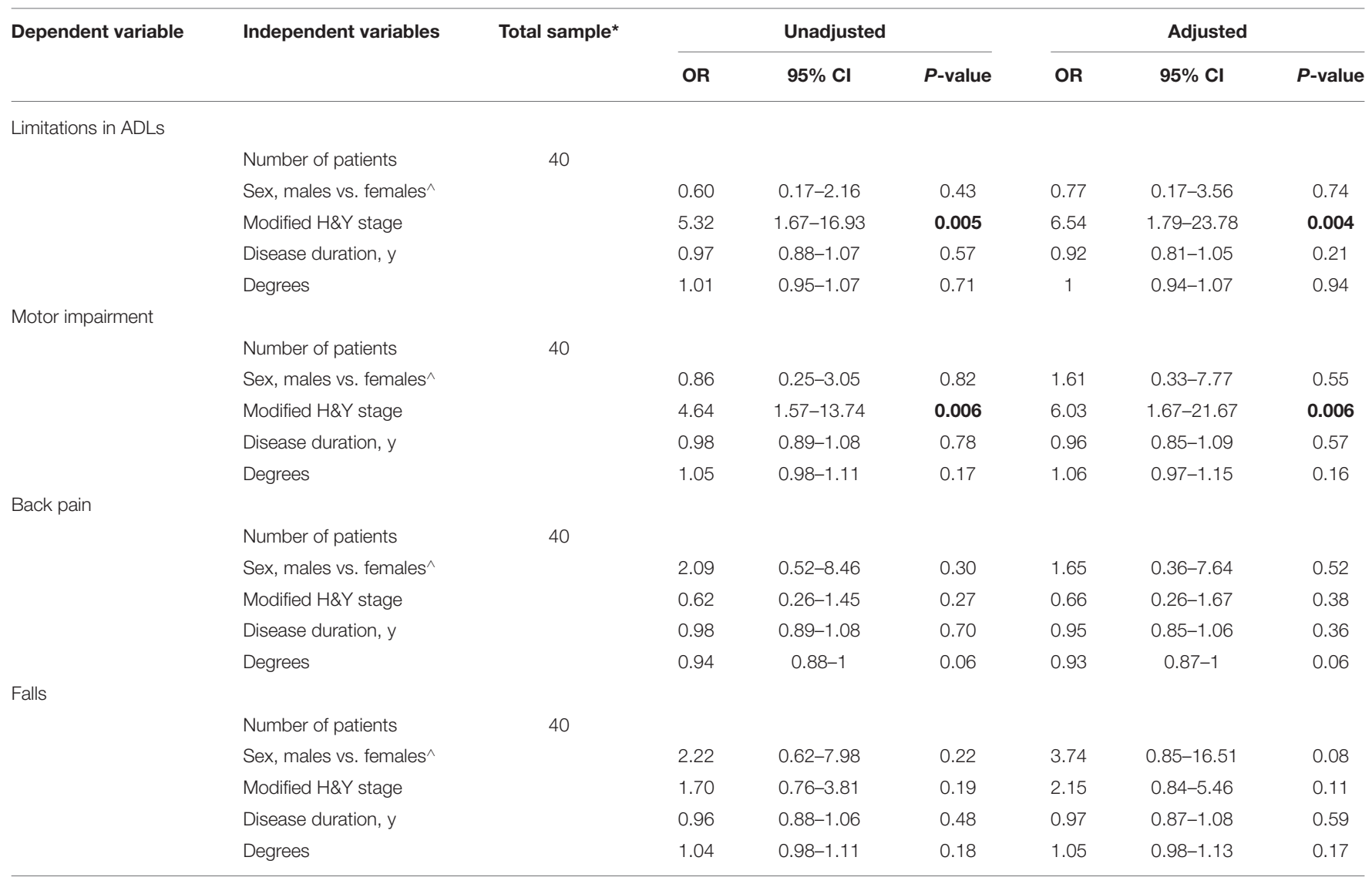

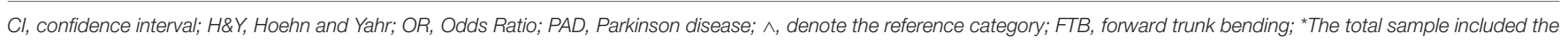
isolated lower FTB $(n=27)$ and combined forms (upper + lower FTB, $n=13$ ); significant associations in bold at $p<0.05$.

LTB and 61 FNB. Upper FTB ranged from $21.3^{\circ}$ to $72.3^{\circ}$ [mean and standard deviation SBM $44.8 \pm 8.6$, WG $41.9^{\circ}$ $\pm 8.9^{\circ}$ ]. Lower FTB ranged from $5^{\circ}$ to $60^{\circ}$ (SBM $28.9^{\circ} \pm$ $10.6^{\circ}$, WG $25.2^{\circ} \pm 12.4^{\circ}$ ). Coexistent upper/lower subtype ranged from $20^{\circ}$ to $60^{\circ}$ (SBM upper $40.7^{\circ} \pm 10.1^{\circ}$, WG $45.8^{\circ} \pm 11.7^{\circ}$; SBM lower $31.7^{\circ} \pm 9.2^{\circ}$, WG $28.1^{\circ} \pm 8.8^{\circ}$ ). $\mathrm{LTB}$ ranged from $5^{\circ}$ to $45^{\circ}\left(\mathrm{SBM} 11.3^{\circ} \pm 7.4^{\circ}\right.$, WG $11.7^{\circ}$ $\pm 7.4^{\circ}$ ). FNB ranged from $15^{\circ}$ to $106.2^{\circ}$ (SBM $62.5^{\circ} \pm$ $20^{\circ}$, WG $58.7^{\circ} \pm 20.4^{\circ}$ ). Table 1 presents the clinical and demographical features.

\section{Correlation of Clinical and Demographical Variables With Limitation in ADLs, Motor Impairment, Falls, and Pain}

In the measures taken with the WG, the univariate logistic regression model yielded significant associations between upper/lower FTB, LTB, and FNB and many of the investigated clinical and demographic features (Tables 2-5). After adjusting for all variables in the model, multivariate logistic regression analysis confirmed the following associations.

Upper FTB: modified H\&Y stage (OR 4.34, 95\% CI 2.47-7.61) and disease duration (OR 1.08, 95\% CI 1-1.17) were associated with limitations in ADLs. The modified H\&Y stage was also associated with motor impairment (OR 2.43, 95\% CI $1.53-$ 3.85 ), back pain (OR 1.63, 95\% CI 1.06-2.49), and falls (OR 2.06, 95\% CI 1.27-3.32) (Table 2).

Lower FTB: modified H\&Y stage was associated with limitations in ADLs (OR 6.54, 95\% CI 1.79-23.78) and motor impairment (OR 6.03, 95\% CI 1.67-21.67) (Table 3).

LTB: modified H\&Y stage (OR 4.26, 95\% CI 2.07-8.77) was associated with limitations in ADLs. Sex (OR 0.35, 95\% CI 0.12-0.99), modified H\&Y stage (OR 2.75, 95\% CI 1.33-5.58), and degree of trunk bending (OR 1.12, 95\% CI 1.03-1.22) were associated with motor impairment. Sex (OR 0.33, 95\% CI 0.130.87 ) and modified H\&Y stage (OR 1.86, 95\% CI 1.01-3.41) were associated with back pain (Table 4).

FNB: modified H\&Y stage (OR 3.37, 95\% CI 1.33-8.53) was associated with motor impairment (Table 5).

The SBM showed similar results in the adjusted and unadjusted ORs. No other statistically significant value was found for upper/lower FTB, LTB, and FNB measured with the WG and SBM (Tables 2-5 and Supplementary Tables 1-4).

\section{ROC Curve Analysis}

Given the significant association in the multivariate logistic regression model, we performed ROC analysis to predict the 
TABLE 4 | Clinical and demographic variables associated with LTB, as measured with a wall goniometer.

\begin{tabular}{|c|c|c|c|c|c|c|c|c|}
\hline Dependent variable & Independent variables & Total sample & \multicolumn{3}{|c|}{ Unadjusted } & \multicolumn{3}{|c|}{ Adjusted } \\
\hline & Number of patients & 88 & & & & & & \\
\hline & Sex, males vs. females ${ }^{\wedge}$ & & 1.08 & $0.46-2.56$ & 0.85 & 0.72 & $0.23-2.23$ & 0.57 \\
\hline & Modified H\&Y stage & & 4.66 & $2.46-8.82$ & $<0.0005$ & 4.26 & $2.07-8.77$ & $<0.0005$ \\
\hline & Degrees & & 1.02 & $0.96-1.08$ & 0.46 & 1.08 & $0.99-1.17$ & 0.07 \\
\hline \multicolumn{9}{|l|}{ Motor impairment } \\
\hline & Number of patients & 88 & & & & & & \\
\hline & Sex, males vs. females^ & & 0.54 & $0.22-1.29$ & 0.16 & 0.35 & $0.12-0.99$ & 0.049 \\
\hline & Modified H\&Y stage & & 2.14 & $1.24-3.70$ & 0.006 & 2.75 & $1.33-5.58$ & 0.005 \\
\hline & Sex, males vs. females^ & & 0.41 & $0.16-0.99$ & 0.049 & 0.33 & $0.13-0.87$ & 0.024 \\
\hline & Modified H\&Y stage & & 1.53 & $0.94-2.94$ & 0.08 & 1.86 & $1.01-3.41$ & 0.046 \\
\hline & Disease duration, y & & 1.02 & $0.96-1.08$ & 0.56 & 0.98 & $0.92-1.06$ & 0.74 \\
\hline & Degrees & & 1.03 & $0.97-1.09$ & 0.38 & 1.05 & $0.98-1.13$ & 0.15 \\
\hline \multicolumn{9}{|l|}{ Falls } \\
\hline & Number of patients & 88 & & & & & & \\
\hline & Sex, males vs. females ${ }^{\wedge}$ & & 1.56 & $0.60-4.02$ & 0.36 & 1.45 & $0.54-3.92$ & 0.46 \\
\hline & Modified H\&Y stage & & 1.61 & $0.93-2.79$ & 0.09 & 1.82 & $0.96-3.44$ & 0.07 \\
\hline & Disease duration, y & & 1.02 & $0.95-1.08$ & 0.59 & 0.99 & $0.92-1.07$ & 0.84 \\
\hline & Degrees & & 1.05 & $0.98-1.11$ & 0.13 & 1.06 & $0.99-1.13$ & 0.08 \\
\hline
\end{tabular}

Cl, confidence interval; H\&Y, Hoehn and Yahr; OR, Odds Ratio; LTB, Lateral Trunk Bending; $\wedge$, denotes the reference category; significant associations in bold at $p<0.05$.

LTB cut-off angle for best discriminating low vs. high disability. According to the clinical WG evaluation, the optimal cut-off value was $12.5^{\circ}$, with a sensitivity of 0.5 and a specificity of 0.8 (AUC $0.615 ; p: 0.071$ ). According to the SBM, the optimal cut-off value was $10.5^{\circ}$, with a sensitivity of 0.5 and a specificity of 0.8 (AUC 0.626; p: 0.048).

\section{DISCUSSION}

With this study we wanted to analyze the influence of trunk bending severity on disability and determine the cut-off values of trunk bending that best discriminated limitations in ADLs, motor impairment, falls, and back pain. The adjusted odds ratios showed a significant association between motor impairment and degree of LTB, indicating that an increase in lateral bending increases the probability of greater motor impairment. We found that the optimal cut-off to identify moderate to severe motor impairment was $10.5^{\circ}$, corresponding to the most common cutoff for the diagnosis of PS $(3,11)$. In contrast, for upper/lower FTB and FNB we found no association between limitation of ADLs, motor impairment, pain, and falls and the degree of bending, except for a more advanced stage of disease (H\&Y stage), longer disease duration, and sex. Our data cannot explain the association between the degree of LTB and female gender with motor impairment. However, we hypothesize that trunk asymmetry related to LTB may reduce trunk mobility more than FTB, leading to a greater impairment in motor functions, and intrinsic gender differences of the anatomy of the spine (24) may be a predisposing factor, along with subclinical osteoporosis for which the frequency in women is more than double that in men (25).

PD-associated postural abnormalities have attracted increasing attention from clinicians and researchers since they were recognized as a frequent and disabling complication of PD (1, 3-6, 26). Most studies on severe forward and lateral trunk flexion (i.e., CC, PS, and AC) to date have applied arbitrary and often diverse diagnostic criteria $(2,3)$. Despite the different criteria employed, greater disability is noted in patients with CC, PS, and AC than in those without severe postural abnormalities (1). Moreover, patients with lower CC seem to have more severe gait and postural control impairment than those with upper CC and without CC (26). Our data show that the severity of trunk bending cannot fully explain impairments. Patients with a more advanced PD stage and longer disease duration are at higher risk of having limitations in ADLs, motor impairment, back pain, and falls, independent of the degree of trunk bending. Our data are consistent with previous studies indicating that the association between postural abnormalities and such variables may be more closely related to the severity of the PD phenotype and disease progression than to the severity of trunk flexion itself $(1,5)$. 
TABLE 5 | Clinical and demographic variables associated with FNB, as measured with a wall goniometer.

\begin{tabular}{|c|c|c|c|c|c|c|c|c|}
\hline Dependent variable & Independent variables & Total sample & \multicolumn{3}{|c|}{ Unadjusted } & \multicolumn{3}{|c|}{ Adjusted } \\
\hline & Number of patients & 61 & & & & & & \\
\hline & Sex, males vs. females ${ }^{\wedge}$ & & 0.93 & $0.30-2.88$ & 0.90 & 1.27 & $0.26-6.18$ & 0.77 \\
\hline & Modified H\&Y stage & & 3.98 & $1.92-8.23$ & $<0.0005$ & 2.32 & $0.97-5.50$ & 0.06 \\
\hline & Degrees & & 1.03 & $1-1.06$ & 0.027 & 1.02 & $0.98-1.05$ & 0.32 \\
\hline \multicolumn{9}{|l|}{ Motor impairment } \\
\hline & Number of patients & 61 & & & & & & \\
\hline & Sex, males vs. females ${ }^{\wedge}$ & & 0.73 & $0.24-2.25$ & 0.58 & 0.93 & $0.24-3.54$ & 0.91 \\
\hline & Modified H\&Y stage & & 2.60 & $1.33-5.10$ & 0.005 & 3.37 & $1.33-8.53$ & 0.010 \\
\hline & Sex, males vs. females ${ }^{\wedge}$ & & 1.87 & $0.56-6.19$ & 0.30 & 1.76 & $0.46-6.73$ & 0.41 \\
\hline & Modified H\&Y stage & & 1.29 & $0.74-2.25$ & 0.36 & 2.16 & $0.93-5.02$ & 0.07 \\
\hline & Disease duration, y & & 0.97 & $0.90-1.04$ & 0.42 & 0.91 & $0.81-1.01$ & 0.08 \\
\hline & Degrees & & 1.01 & $0.98-1.04$ & 0.33 & 1.01 & $0.98-1.04$ & 0.57 \\
\hline \multicolumn{9}{|l|}{ Falls } \\
\hline & Number of patients & 61 & & & & & & \\
\hline & Sex, males vs. females ${ }^{\wedge}$ & & 2.68 & $0.67-10.74$ & 0.16 & 4.69 & $0.98-22.35$ & 0.05 \\
\hline & Modified H\&Y stage & & 1.04 & $0.57-1.86$ & 0.90 & 0.85 & $0.35-2.07$ & 0.72 \\
\hline & Disease duration, y & & 1.03 & $0.96-1.10$ & 0.41 & 1.07 & $0.96-1.20$ & 0.18 \\
\hline & Degrees & & 0.98 & $0.96-1.02$ & 0.39 & 0.97 & $0.94-1.01$ & 0.11 \\
\hline
\end{tabular}

Cl, confidence interval; H\&Y, Hoehn and Yahr; OR, Odds Ratio; FNB, Forward Neck Bending; $\wedge$, denotes the reference category; $y$, years; significant associations in bold at $p<0.05$.

With regard to limitations in ADLs and perceived pain, a study uncorrected for possible confounders involving 145 subjects with PD and FTB did not yield convincing evidence for a threshold angle defining CC (27). Indeed, while showing a significant association between FTB $\geq 30^{\circ}$ and limitations in ADLs and back pain, the authors remarked that less severe forward angles do not exclude CC (27). They found greater limitations in ADLs in patients with FTB $\geq 30^{\circ}$, which is in line with our univariate regression models reported for upper FTB. Nevertheless, here we demonstrate that this association did not survive multivariate logistic regression analysis, which indicated that sex, duration and stage of disease are relevant confounding factors.

Pain, one of the most frequent non-motor symptoms of PD, has been strongly associated with a worse quality of life (2830). The relationship between PD and pain is complex and not fully elucidated; however, it has been demonstrated that PD patients with motor complications have a greater risk of developing pain (31), which seems to increase with worsening parkinsonian symptoms (28). Our findings suggest that the back pain in patients with trunk misalignment could be more closely related to the PD stage and duration than to the postural alterations.

Hence, the severity of trunk/neck bending alone does not explain limitation in ADLs, motor impairment, falls, and back pain in PD patients. Multiple factors possibly related to an aggressive PD phenotype account for disability. Nonetheless, less severe degrees of trunk bending-though not classified as CC, PS, or AC-should be monitored (i.e., quantitively by using apps) (32) and promptly treated (i.e., physiotherapy) (33) to prevent progression and worsening and to avoid permanent deformity. Physiotherapy should include active selfcorrection, trunk stabilization exercises (i.e., strengthening of compensatory paraspinal muscles) and functional tasks, along with pharmacological intervention such as botulinum toxin injection to reduce muscle hyperactivity $(4,14,33,34)$.

Some limitations should be taken into account when interpreting our results. First, the ROC analysis showed only a moderate accuracy of discrimination, as reflected by AUC values and the not very high values of sensitivity and specificity. Second, the necessity of a dichotomous outcome to run the ROC analysis required us to identify validated cut-offs for the patient-centered outcome measures. We could not find such cut-off values for Parkinson's disease Questionnaire 8, which would have extended the analysis to include quality of life. Third, the history of falls was based on patient-reported number of falls in the month before assessment, and no significant FTB, LTB, or FNB values were found for discriminating the history of falls. It is possible that analysis with more accurate recording of falls might have 
yielded significant results. Fourth, we did not evaluate as specific outcomes motor fluctuations, cognitive impairment, and freezing of gait which can be present in patients with $\mathrm{PD}$ and postural abnormalities (35) and associated with poor quality of life and falls. Finally, a prospective study design would help clarify the association and risk factors for disability in a cohort of patients with postural abnormalities.

These limitations notwithstanding, our findings indicate that advanced stage of disease and longer disease duration, but not the degree of trunk bending, may explain the greater disability of PD patients with postural abnormalities. Limitation in ADLs and presence history of falls and severe pain in FTB, LTB, and FNB may be more closely related to PD phenotype and progression than trunk flexion itself $(1,5)$. In contrast, moderate/severe motor impairment can be predicted by a degree of LTB $>10^{\circ}$, which is the cut-off typically used for the diagnosis of PS. This is an important finding because it justifies the widely used cut-off of $10^{\circ}$ defined a priori by previous studies. Summarizing, all patients with PS should be referred to management by a multidisciplinary team at an early stage to improve their quality of life and slow the progress of motor decline (4).

\section{ETHICS STATEMENT}

The studies involving human participants were reviewed and approved by Azienda Ospedaliera Universitaria

\section{REFERENCES}

1. Tinazzi M, Gandolfi M, Ceravolo R, Capecci M, Andrenelli E, Ceravolo MG, et. al. Postural abnormalities in Parkinson's disease: an epidemiological and clinical multicenter study. Mov Disord Clin Pract. (2019) 6:576-85. doi: $10.1002 / \mathrm{mdc} 3.12810$

2. Fasano A, Geroin C, Berardelli A, Bloem BR, Espay AJ, Hallett M, et al. Diagnostic criteria for camptocormia in Parkinson's disease: a consensus-based proposal. Parkinsonism Relat Disord. (2018) 53:53-7. doi: 10.1016/j.parkreldis.2018.04.033

3. Doherty KM, van de Warrenburg BP, Peralta MC, Silveira-Moriyama L, Azulay JP, Gershanik OS, et al. Postural deformities in Parkinson's disease. Lancet Neurol. (2011) 10:538-49. doi: 10.1016/S1474-4422(11)70067-9

4. Tinazzi M, Geroin C, Gandolfi M, Smania N, Tamburin S, Morgante F, et al. Pisa syndrome in Parkinson's disease: an integrated approach from pathophysiology to management. Mov Disord. (2016) 31:1785-95. doi: $10.1002 / \mathrm{mds} .26829$

5. Srivanitchapoom P, Hallett M. Camptocormia in parkinson's disease: definition, epidemiology, pathogenesis and treatment modalities. J Neurol Neurosurg Psychiatry. (2016) 87:75-85. doi: 10.1136/jnnp-2014-310049

6. Pandey S, Kumar H. Assessment of striatal \& postural deformities in patients with Parkinson's disease. Indian J Med Res. (2016) 144:682-8. doi: 10.4103/ijmr.IJMR_502_15

7. Postuma RB, Berg D, Stern M, Poewe W, Olanow CW, Oertel W, et al. MDS clinical diagnostic criteria for Parkinson's disease. Mov Disord. (2015) 30:1591-601. doi: 10.1002/mds.26424

8. Movement disorder society task force on rating scales for Parkinson's disease. The unified Parkinson's disease rating scale (UPDRS): status and recommendations. Mov Disord. (2003) 18:738-50. doi: 10.1002/mds.10473

9. Foltynie T, Brayne C, Barker RA. The heterogeneity of idiopathic Parkinson's disease. J Neurol. (2002) 249:138-45. doi: 10.1007/PL00007856
Integrata Verona. The patients/participants provided their written informed consent to participate in this study. Written informed consent was obtained from the individual(s) for the publication of any potentially identifiable images or data included in this article.

\section{AUTHOR CONTRIBUTIONS}

CG, CA, and MT drafting and revising the manuscript, study concept and design, acquisition, analysis and interpretation of data, and study execution. MG, EZ, and RC drafting/revising the manuscript, study concept and design, interpretation of data, and study execution. MCap, EA, MGC, LB, MO, RT, GB, MCat, PM, SM, SG, LV, FS, MCas, CF-P, MZ, AF, and LL drafting/revising the manuscript, acquisition and interpretation of data, and study execution.

\section{FUNDING}

This work was supported by FONDAZIONE Cariverona.

\section{SUPPLEMENTARY MATERIAL}

The Supplementary Material for this article can be found online at: https://www.frontiersin.org/articles/10.3389/fneur. 2020.00207/full\#supplementary-material
10. Tinazzi M, Gandolfi M, Artusi CA, Lanzafame R, Zanolin E, Ceravolo $\mathrm{R}$, et al. Validity of the wall goniometer as a screening tool to detect postural abnormalities in Parkinson's disease. Parkinsonism Relat Disord. (2019) 69:159-65. doi: 10.1016/j.parkreldis.2019. 10.024

11. Tinazzi M, Fasano A, Geroin C, Morgante F, Ceravolo R, Rossi S, et al. Italian pisa syndrome study group. Pisa syndrome in Parkinson disease: an observational multicenter Italian study. Neurology. (2015) 85:1769-79. doi: 10.1212/WNL.0000000000002122

12. Littrell ME, Chang YH, Selgrade BP. Development and assessment of a low-cost clinical gait analysis system. J Appl Biomech. (2018) 10:1-19. doi: 10.1123/jab.2017-0370

13. Margraf NG, Wolke R, Granert O, Berardelli A, Bloem BR, Djaldetti $\mathrm{R}$, et al. Consensus for the measurement of the camptocormia angle in the standing patient. Parkinsonism Relat Disord. (2018) 52:1-5. doi: 10.1016/j.parkreldis.2018.06.013

14. Artusi CA, Bortolani S, Merola A, Zibetti M, Busso M, De Mercanti $S$, et al. Botulinum toxin for pisa syndrome: an MRI-, ultrasound- and electromyography-guided pilot study. Parkinsonism Relat Disord. (2019) 62:231-5. doi: 10.1016/j.parkreldis.2018.11.003

15. Grimmer-Somers K, Milanese S, Louw Q. Measurement of cervical posture in the sagittal plane. J Manipulative Physiol Ther. (2008) 31:509-17. doi: 10.1016/j.jmpt.2008.08.005

16. Shulman LM, Armstrong M, Ellis T, Gruber-Baldini A, Horak F, Nieuwboer A, et al. Disability rating scales in Parkinson's disease: critique and recommendations. Mov Disord. (2016) 31:1455-65. doi: 10.1002/mds.26649

17. Rodriguez-Blazquez C, Rojo-Abuin JM, Alvarez-Sanchez M, Arakaki T, Bergareche-Yarza A, et al. The MDS-UPDRS Part II (motor experiences of daily living) resulted useful for assessment of disability in Parkinson's disease. Parkinsonism Relat Disord. (2013) 19:889-93. doi: 10.1016/j.parkreldis.2013.05.017 
18. Martínez-Martín P, Rodríguez-Blázquez C, Alvarez M, Arakaki T, Arillo VC, Chaná P, et al. Parkinson's disease severity levels and MDS-Unified Parkinson's disease rating scale. Parkinsonism Relat Disord. (2015) 21:50-4. doi: $10.1016 /$ j.parkreldis.2014.10.026

19. The prevention of falls in later life. A report of the kellogg international work group on the prevention of falls by the elderly. Dan Med Bull. (1987) 34(Suppl. 4):1-24.

20. Perez-Lloret S, Ciampi de Andrade D, Lyons KE, Rodríguez-Blázquez C, Chaudhuri KR, Deuschl G, et al. Members of the MDS committee on rating scales development. Rating scales for pain in Parkinson's disease: critique and recommendations. Mov Disord Clin Pract. (2016) 3:527-37. doi: $10.1002 / \mathrm{mdc} 3.12384$

21. Müller T, Muhlack S, Woitalla D. Pain perception, pain drug therapy and health status in patients with Parkinson's disease. Neuroepidemiology. (2011) 37:183-7. doi: 10.1159/000331911

22. Hartrick CT, Kovan JP, Shapiro S. The numeric rating scale for clinical pain measurement: a ratio measure? Pain Pract. (2003) 3:310-6. doi: 10.1111/j.1530-7085.2003.03034.x

23. Fischer JE, Bachmann LM, Jaeschke R. A readers' guide to the interpretation of diagnostic test properties: clinical example of sepsis. Intensive Care Med. (2003) 29:1043-51. doi: 10.1007/s00134-003-1761-8

24. Marras WS, Jorgensen MJ, Granata KP, Wiand B. Female and male trunk geometry: size and prediction of the spine loading trunk muscles derived from MRI. Clin Biomech. (2001) 16:38-46. doi: 10.1016/S0268-0033(00)00046-2

25. Alswat KA. Gender disparities in osteoporosis. J Clin Med Res. (2017) 9:382-7. doi: 10.14740/jocmr2970w

26. Geroin C, Gandolfi M, Smania N, Maddalena I, Tinazzi M. Do upper and lower camptocormias affect gait and postural control in patients with Parkinson's disease: an observational cross-sectional study. Parkinsons Dis. (2019) 2019:9026890. doi: 10.1155/2019/9026890

27. Margraf NG, Granert O, Hampel J, Wrede A, Schulz-Schaeffer WJ, Deuschl G. Clinical definition of camptocormia in Parkinson's disease. Mov Disord Clin Pract. (2016) 4:349-57. doi: 10.1002/mdc3.12437

28. Defazio G, Gigante A, Mancino P, Tinazzi M. The epidemiology of pain in parkinson's disease. J Neural Transm. (2013) 120:583-6. doi: 10.1007/s00702-012-0915-7

29. Geroin C, Gandolfi M, Bruno V, Smania N, Tinazzi M. Integrated approach for pain management in parkinson disease. Curr Neurol Neurosci Rep. (2016) 16:28. doi: 10.1007/s11910-016-0628-7
30. Gandolfi M, Geroin C, Antonini A, Smania N, Tinazzi M. Understanding and treating pain syndromes in parkinson's disease. Int Rev Neurobiol. (2017) 134:827-58. doi: 10.1016/bs.irn.2017. 05.013

31. Tinazzi M, Del Vesco C, Fincati E, Ottaviani S, Smania N, Moretto G, et al. Pain and motor complications in Parkinson's disease. J Neurol Neurosurg Psychiatry. (2006) 77:822-5. doi: 10.1136/jnnp.2005.0 79053

32. Schlenstedt C, Boße K, Gavriliuc O, Wolke R, Granert O, Deuschl $\mathrm{G}$, et al. Quantitative assessment of posture in healthy controls and patients with Parkinson's disease. Parkinsonism Relat Disord. (2020) doi: 10.1016/j.parkreldis.2020.01.012

33. Geroin C, Squintani G, Morini A, Donato F, Smania N, Gandolfi MG, et al. Pisa syndrome in Parkinson's disease: electromyographic quantification of paraspinal and non-paraspinal muscle activity. Funct Neurol. (2017) 32:14351. doi: 10.11138/FNeur/2017.32.3.143

34. Gandolfi M, Tinazzi M, Magrinelli F, Busselli G, Dimitrova E, Polo N, et al. Four-week trunk-specific exercise program decreases forward trunk flexion in parkinson's disease: a single-blinded, randomized controlled trial. Parkinsonism Relat Disord. (2019) 64:268-74. doi: 10.1016/j.parkreldis.2019. 05.006

35. Artusi CA, Montanaro E, Tuttobene S, Romagnolo A, Zibetti M, Lopiano L. Pisa syndrome in parkinson's disease is associated with specific cognitive alterations. Front Neurol. (2019) 10:577. doi: 10.3389/fneur.2019. 00577

Conflict of Interest: The authors declare that the research was conducted in the absence of any commercial or financial relationships that could be construed as a potential conflict of interest.

Copyright (C) 2020 Geroin, Artusi, Gandolfi, Zanolin, Ceravolo, Capecci, Andrenelli, Ceravolo, Bonanni, Onofrj, Telese, Bellavita, Catalan, Manganotti, Mazzucchi, Giannoni, Vacca, Stocchi, Casali, Falup-Pecurariu, Zibetti, Fasano, Lopiano and Tinazzi. This is an open-access article distributed under the terms of the Creative Commons Attribution License (CC BY). The use, distribution or reproduction in other forums is permitted, provided the original author(s) and the copyright owner(s) are credited and that the original publication in this journal is cited, in accordance with accepted academic practice. No use, distribution or reproduction is permitted which does not comply with these terms 